\title{
Analysis of Acoustic and Spray Combine Agglomeration to Removal of Coal-fired Fly Ash Fine Particles
}

\author{
Jie Wang ${ }^{1}$, Jianzhong Liu $^{2}$ \\ 1.Zhejiang Energy Group R \& D, Zhejiang University, Hangzhou, Zhejiang, China \\ 2.Zhejiang University, Hangzhou, Zhejiang, China
}

Keywords: Coal-fired fly ash; Fine particles; Acoustic and spray combine agglomeration.

\begin{abstract}
Particulate pollution is a major kind of atmospheric pollution. The fine particles are seriously harmful to human health and environment. Acoustic agglomeration has been proved as a promising pretreatment to control the emission of fine particles. Based on the acoustic agglomeration, this paper investigates the acoustic and spray combine agglomeration. The typical experiment reveals that spray agglomeration can significantly improve acoustic agglomeration effect, the improvement of agglomeration efficiency can achieve $40 \%$.The influence of frequency, SPL, spray flow and spray angle on agglomeration efficiency are analyzed.
\end{abstract}

\section{INTRODUCTION}

Particulate pollution is seriously harmful to human health and environment and has raised a great concern in international community. Particulate pollution is mainly caused by the burning of fossil fuels, vehicle exhaust emissions, steel industry production and human activities [Bi Xiaohui, 2007]. The particle with aerodynamic diameter less than $2.5 \mu \mathrm{m}$ (PM2.5) can enter into alveoli, even deeply into the capillaries, and cause greater damage to human body [Lewtas, 2007].

The acoustic agglomeration is one of the most promising pretreatment technologies. The combination of acoustic agglomeration with conventional particle filtering devices makes it easy to remove fine particles from the flue gas [Zhang Guangxue, 2009][Shuster, 2002]. Based on the lowfrequency sound source [Liu Jianzhong, 2011], by wetting the fly ash aerosol, acoustic and spray combine agglomeration can significantly improve the aggregation efficiency. Due to agglomeration process is affected by a variety of parameters and unknown factors, the studies have not come to uniform conclusion.

\section{EXPERIMENTAL ARRANGEMENT}

As shown in Figure 1, the acoustic agglomeration system consists of agglomeration chamber, sound source, fly ash feeding, spray system, aerosol sampling and measurement.

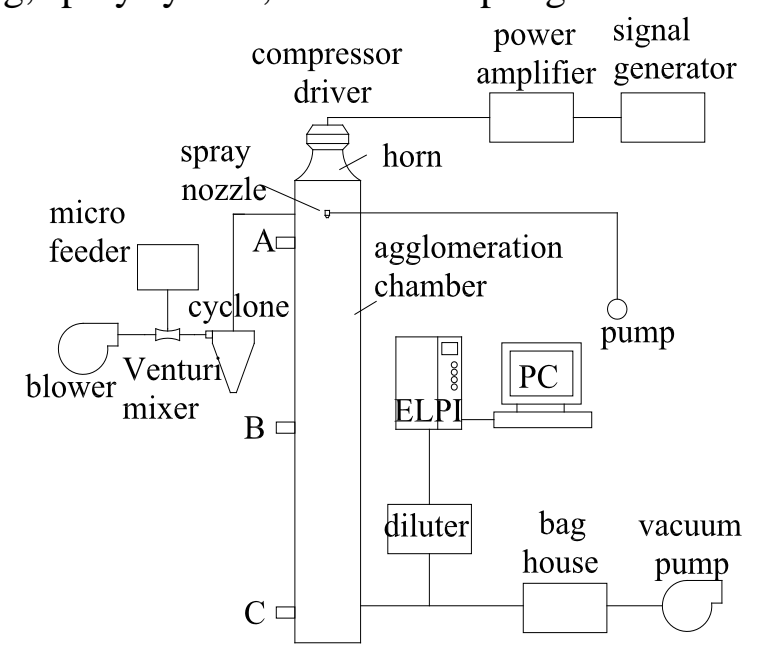

Figure 1. Geometry of centrifugal pump and grid. 
The agglomeration chamber is made of a vertical tube. Sound source is made up of a horn and compression driver and installed on the top of the chamber. The compression driver is combined with an amplifier, which is powered by a signal generator. The spray system contains a nozzle and plunger pump, and the spray flow and angle can be adjusted. Three acoustic measurement points, point $\mathrm{A}, \mathrm{B}$ and $\mathrm{C}$ are set along the chamber wall at regular intervals.

The coal-fired fly ash particles are collected from an electrostatic precipitator of coal-fired boiler, which are continuously given by the micro feeder are mixed with clean air-stream in the Venturi mixer. Coarse particles in the initial aerosol are removed by a cyclone with a cut-diameter of $10 \mu \mathrm{m}$. Then the aerosol enters into the agglomeration chamber. At the outlet of the chamber an electrical low pressure impactor (ELPI) is used to measure the aerosol real-time PSDs with 12 channels.

All experiments are carried out at ambient temperature, i.e. $25{ }^{\circ} \mathrm{C}$, and all the measurements are performed after the system is operated steadily for more than $10 \mathrm{~min}$.

\section{Results and analysis}

\section{Theoretical analysis}

The mechanisms peculiar to the acoustic agglomeration are very complex. The local acoustic velocity ui can be calculated by

$$
u_{i}=\operatorname{Re}\left(U_{0} e^{-j \omega t}\right)
$$

Where, $U_{0}=$ velocity amplitude of the gas medium, $\omega=$ angular frequency, $t=$ time. Assuming $U_{0}$ is much smaller than the sound speed, and the particle diameter, $d_{\mathrm{p}}$, is much smaller than the acoustic wavelength, the particle oscillating velocity, $u_{p}$, can be calculated by

$$
u_{p}=\operatorname{Re}\left(H U_{0} e^{-j \omega t}\right)
$$

Where, $H=$ complex entrainment function, The acoustic entrainment factor, $\mu_{\mathrm{p}}$, which is defined as the ratio of particle amplitude to gas amplitude in the sound wave, can be calculated by

$$
\mu_{p}=\frac{u_{p 0}}{u_{0}}=\frac{1}{\sqrt{1+\left(\omega \tau_{d}\right)^{2}}}
$$

Where, $\tau_{\mathrm{d}}=$ particle dynamic relaxation time, given by [Tiwary and Reethof, 1987]

$$
\tau_{d}=\frac{\rho_{p} d_{p}{ }^{2}}{18 \mu}
$$

Where, $\rho_{\mathrm{p}}=$ particle density, $\mu=$ dynamic viscosity of the gas medium. Combining equations can find that $\mu_{\mathrm{p}}$ is related to many factors. While in the acoustic agglomeration $\mu$ and $\rho_{\mathrm{p}}$ are considered as constants, so the entrainment factor is mainly related to the frequency and $d_{\mathrm{p}}$.

When the mist is sprayed into the coal-fired fly ash aerosol, the droplets spread on the particle surface. Liquid bridge force is formed on the surface of individual particles, when all the particles are wetted to form the liquid bridge force. The size of aggregate particle is recognized as critical particle size. If the mist spray continues, a gap is formed between the particles, and the agglomerated poriferous particle is formed. When the humidity continues to increase, due to the capillary action the poriferous particle is filled with liquid and becomes saturated. Then the liquid force between the agglomerated particles is formed, which promotes to form a bigger poriferous particle. Humidity further increases, the bigger poriferous particles are completely filled and uniformly disperse in the mist, the agglomeration process is completed.

Only considering the adherence effect of mist, the agglomeration model can be simplified. According to Young equation, the contact angle $\theta$ can be calculated as:

$$
\cos \theta=\frac{\gamma_{G-S}-\gamma_{L-S}}{\gamma_{G-L}}
$$

Where, $\gamma_{-S}=$ surface tension between the surfaces, subscripts of $G, L$ and $S$ represent gas, liquid and solid. If the difference between $\gamma_{G \text {-S }}$ and $\gamma_{L-S}$ is greater, the contact angle is smaller. If the contact angle is $0^{\circ}$, the droplet can be considered completely spread on the solid surface and the agglomeration can be quicker and better. 


\section{Typical experimental results}

The experimental study of combined agglomeration is based on the low frequency acoustic agglomeration, wetting the coal fly ash aerosol is applied. The agglomeration efficiencies are compared to analyze the efficiency improvement of spray. Initial particle number concentration is expected to decrease. The agglomeration efficiencies are calculated by

$$
\begin{aligned}
& \eta_{1}=\left(1-\frac{N_{1}}{N_{0}}\right) \times 100 \% ; \quad \eta_{1}{ }^{\prime}=\left(1-\frac{N_{1}{ }^{\prime}}{N_{0}}\right) \times 100 \% ; \\
& \eta_{2}=\left(1-\frac{N_{2}}{N_{0}}\right) \times 100 \% \\
& \Delta \eta=\eta_{2}-\eta_{1} ; \quad \Delta \eta^{\prime}=\eta_{2}-\eta_{1}{ }^{\prime}
\end{aligned}
$$

Where, $\mathrm{N}=$ particle number concentration, subscripts $0,1,1$ ' and 2 represent the initial, acoustic agglomeration alone, spray agglomeration alone and combination agglomeration of sound and spray. $\Delta \eta, \Delta \eta$ ' are introduced to investigate the improvement of application of sound or spray.

Figure 2 shows the typical aerosol PSDs of the initial, after acoustic agglomeration alone, combination agglomeration, with the frequency of $1400 \mathrm{~Hz}$, SPL of $145 \mathrm{~dB}$, and residence time of 5 s. The initial particle concentration of coal fly ash aerosol is $3.63 \times 105 \mathrm{~cm}-3$.

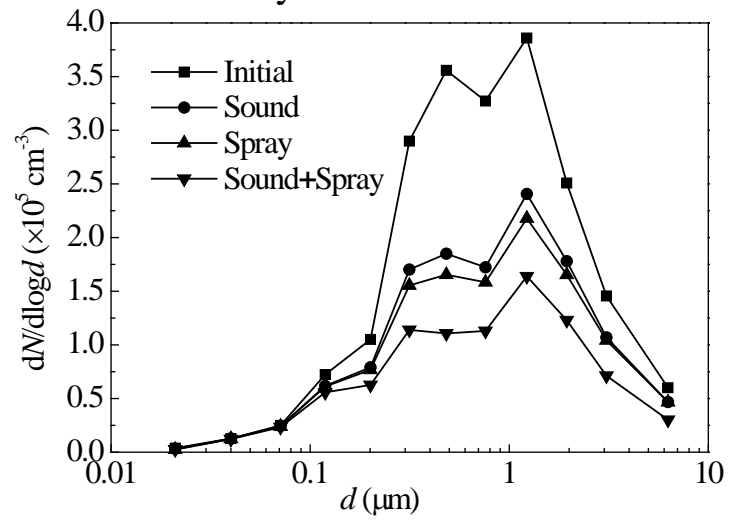

Figure 2. PSDs of initial, with sound alone, spray alone and combination of sound and spray.

The result shows that within the measurement range all the particle concentrations are decreased. According to the calculation results the agglomeration efficiencies of $\eta 1$, $\eta$ ' 1 and $\eta 2$ are $24.68 \%$, $32.80 \%$ and $40.36 \%$, the efficiency improvements $\Delta \eta, \Delta \eta$ ' are $15.68 \%$ and $7.56 \%$. It proves that the combine agglomeration can both improve acoustic agglomeration and spray agglomeration.

Based on previous experimental and theoretical analyses [Wang Jie, 2011], many factors have influences on the agglomeration efficiency. The initial concentration of coal fly ash aerosol is constant to minimize system error. The impact of residence time is small, so it is kept as the best state of 4 second. Keeping the other factors constant, the impact of frequency, SPL, spray flow and angle are analyzed.

\section{Influence of frequency}

Figure 3 shows the influence of frequency with initial aerosol fly ash concentration of $3.62 \times 105$ /cm3, SPL of $140 \mathrm{~dB}$, residence time of 4 second, and spray flow of $0.5 \mathrm{~L} / \mathrm{h}$. 


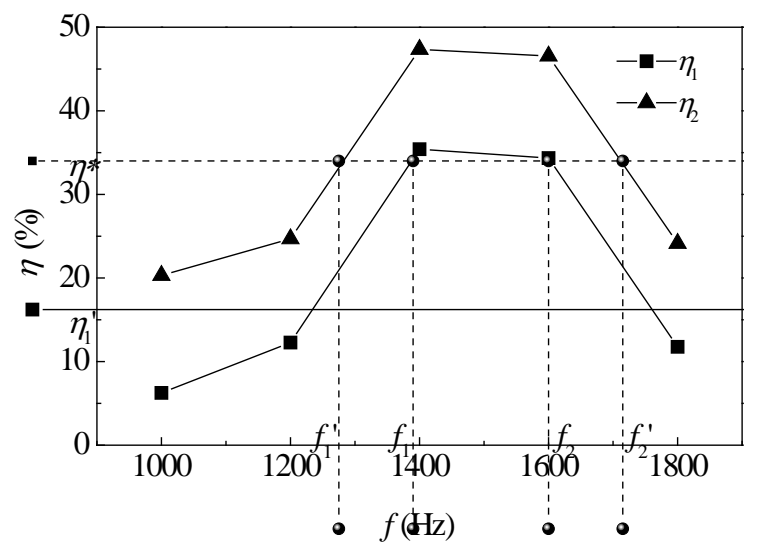

Figure 3. Influence of frequency.

As seen in Figure 4, the optimal frequencies both are $1400 \mathrm{~Hz}$ for $\eta 1$ and $\eta 2$, so $1400 \mathrm{~Hz}$ is the optimum frequency. With the application of spray, $\Delta \eta$ is approximately $12 \% \sim 14 \%$, which changes little with frequency. With the frequency of $1000 \mathrm{~Hz}$, although $\eta 1$ is less, $\Delta \eta$ is highest of $14.06 \%$. Compared to $\eta 1$ ', the improvements of $\eta 1$ and $\eta 2$ are more obvious. Supposing the agglomeration efficiency is needed to reach $\eta^{*}$ in the practical application, with the application of sound alone the selectable range of frequency is $|\mathrm{f} 2-\mathrm{f} 1|$, after the application of spray, the selectable range is widened to $\mid \mathrm{f} 2$ ' - $\mathrm{f} 1^{\prime} \mid$. That means spray agglomeration widens the frequency window of acoustic agglomeration and a better agglomeration could be obtained in a wider frequency range.

Influence of SPL

Figure 4 shows the influence of SPL, with initial aerosol fly ash concentration of $3.68 \times 105 / \mathrm{cm} 3$, frequency of $1400 \mathrm{~Hz}$, residence time of 4 second, spray flow of $2.6 \mathrm{~L} / \mathrm{h}$.

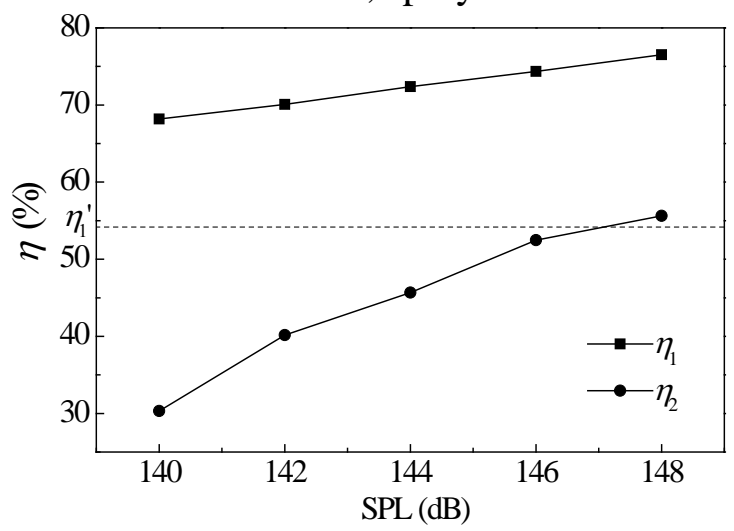

Figure 4. Influence of SPL.

The result shows $\eta 1^{\prime}$ is $54.17 \%$, and it is obvious that the agglomeration effects are better as the SPL becomes higher. When SPL becomes from $140 \mathrm{~dB}$ to $148 \mathrm{~dB}, \eta 1$ and $\eta 2$ improve from $30.33 \%$ and $68.16 \%$ to $55.62 \%$ and $76.51 \%$, which proves that the larger SPL improves the collision between the particles. When SPL is lower, $\eta 1$ is lower, after the application of spray, the agglomeration effect is much better and $\Delta \eta$ is much higher. As SPL becomes higher, $\eta 1$ is higher, but the improvement of agglomeration after the application of spray becomes less. It indicates that the acoustic and spray combine agglomeration has a better effect at lower SPL and the reduction of energy consumption can be expected.

\section{Influence of spray flow}

Figure 5 shows the influence of spray flow, with initial aerosol fly ash concentration of $3.68 \times 105$ /cm3, frequency of $1400 \mathrm{~Hz}$, SPL from 140 to $148 \mathrm{~dB}$, residence time of 4 second. 


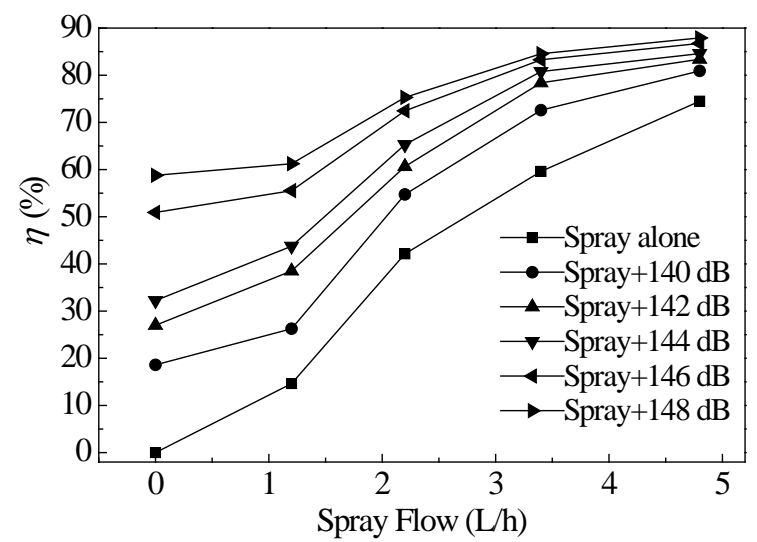

Figure 5. Influence of spray flow.

It shows that the spray flow has a major impact on the combine agglomeration. Enlarging the spray flow with the same of other conditions, the agglomeration efficiency can be obviously improved and the highest agglomeration improvement is $66.33 \%$. When the spray flow is lower, SPL has a greater impact on the combine agglomeration; when the spray flow is larger, the influence of SPL is less, while the agglomeration efficiency keep at higher level about $75 \%$ to $88 \%$.

Influence of spray angle

Figure 6 shows the influence of spray angle, with initial aerosol fly ash concentration of $3.68 \times 105 / \mathrm{cm} 3$, frequency from 1000 to $1800 \mathrm{~Hz}$, residence time of 4 second, and spray flow from 1.2 to $4.8 \mathrm{~L} / \mathrm{h}$.

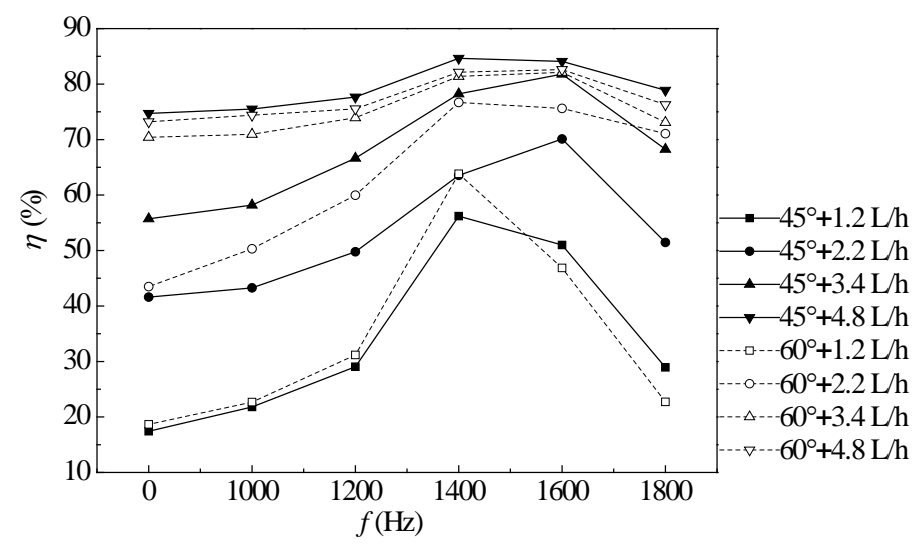

Figure 6. Influence of spray angle.

Figure 6 shows that when the spray flow is lower from 1.2 to $3.4 \mathrm{~L} / \mathrm{h}$, the combine agglomeration efficiency with the spray angle of $60^{\circ}$ is better. When the spray flow is lower, as the jet range is shorter, the larger spray angle can get a better atomization effect, promote the collision between the droplets and coal fly ash particles and get a better agglomeration effect. When the spray flow is higher, with the smaller spray angle the jet range is longer, which make the droplets collide with the coal fly ash particles within longer distance in the chamber and the agglomeration efficiency be better. So in the practical application, when the spray flow is lower, choosing the nozzle with larger spray angle can make the droplets atomize better and get a better agglomeration effect; when the spray flow is higher, choosing the nozzle with smaller spray angle can extend the collision time between the droplets and coal fly ash particles.

\section{Conclusion}

The experimental study proves the spray agglomeration can obvious improve the acoustic agglomeration effect and the improvement can achieve 40\%. The influences of frequency, SPL, spray flow and spray angle are analyzed.

The results prove that the combined acoustic and spray agglomeration can expand the "frequency window", a better agglomeration is obtained in a wider frequency range and the agglomeration 
performance is improved. The application of spray makes the sound source have a better agglomeration effect at lower SPL and reduce the energy consumption of acoustic agglomeration. The larger spray flow can get a better combine agglomeration effect. The influence of spray angle is complicated. When the spray flow is lower, the nozzle with larger spray angle can make the droplets atomize better and get a better agglomeration effect; when the spray flow is higher, the nozzle with smaller spray angle can extend the collision time between the droplets and coal fly ash particles and the agglomeration effect is better.

\section{Acknowledgments}

The authors gratefully acknowledge the kind support of the National Natural Science Foundation of China (50576083), Program New Century Excellent Talents University (NCET-04-0533). The corresponding author is Jie Wang, Emai: wangjieorange@163.com.

\section{REFERENCES}

1. Bi Xiaohui et al. 2007. Source apportionment of PM10 in six cities of northern China. Atmospheric Environment, 41: 903-912.

2. Lewtas, Joellen. 2007. Air pollution combustion emissions: Characterization of causative agents and mechanisms associated with cancer, reproductive, and cardiovascular effects. Mutation Research/Reviews in Mutation Research, 636: 95-133.

3. Zhang Guangxue et al. 2009. Simulation and analysis of collision efficiency in acoustic agglomeration. Journal of Chemical Industry and Engineering(China), 60:42-47.

4. Shuster, K. et al. 2002. Agglomeration of submicrometer particles in weak periodic shock waves. Physics of Fluids, 14:1802-1805.

5. Liu Jianzhong et al. 2011. Frequency comparative study of coal-fired fly ash acoustic agglomeration. Journal of Environmental Sciences, 23: 1845-1851.

6. Wang Jie et al. 2011. Orthogonal design process optimization and single factor analysis for bimodal acoustic agglomeration. Powder Technology, 210:315-322. 